\section{Current practices and intention to provide alcohol-related health advice in primary dental care}

\author{
S. Shepherd, ${ }^{1}$ D. Bonnetti, ${ }^{2}$ J. E. Clarkson, ${ }^{3}$ G. R. Ogden ${ }^{4}$ and L. Young ${ }^{5}$ \\ VERIFIABLE CPD PAPER
}

IN BRIEF
The delivery of alcohol-related health
advice to patients is advocated as one
measure to moderate alcohol consumption.
- Highlights that GDPs are in an ideal
position to identify excessive alcohol
consumption and offer advice.
Posits that beliefs derived from
psychological models may be helpful in
understanding this behaviour and may
provide targets for an intervention to
encourage behaviour change.

\begin{abstract}
Objectives To determine whether general dental practitioners (GDPs) currently provide alcohol-related advice (ARA) and to inform the development of an intervention, should one be required. Method Cross-sectional postal survey of a random sample of 300 GDPs in Scotland. The questionnaire assessed beliefs derived from psychological models that explain behaviour in terms of beliefs that are amenable to change, and so may inform development of an intervention to encourage the provision of ARA. Results Sixty percent of GDPs responded. Eighty-three percent of participating GDPs (145/175) had not provided ARA to patients in the previous ten working days. Attitude (perceived consequences), control beliefs (perceived difficulty), subjective norm (perceived social pressure), and self-efficacy (confidence) significantly predicted intention to provide ARA. Alcohol-related knowledge or personal alcohol behaviour did not predict intention to provide ARA. Conclusions There is scope to increase the provision of ARA in primary care dentistry and this study identified predictive beliefs, which could be targeted to encourage this behaviour. The next phase is to develop and test an intervention to encourage GDPs to provide ARA.
\end{abstract}

\section{BACKGROUND}

Alcohol is responsible for $4.0 \%$ of the global burden of disease and is directly related to a multitude of adverse health conditions. ${ }^{1-4}$ In Scotland alone, alcoholrelated health issues account for National Health Service financial expenditure in the order of hundreds of millions of pounds each year. ${ }^{5-7}$

Evidence suggests that the provision of alcohol-related advice (ARA) by healthcare professionals in primary care could help moderate alcohol consumption. ${ }^{8-13}$

Recently, the Scottish Intercollegiate Guidelines Network and the National

\footnotetext{
1"Lecturer in Oral Surgery, ${ }^{4}$ Professor of Oral Surgery, Unit of Oral Surgery and Medicine, University of Dundee Dental Hospital and School, Park Place, Dundee, DD1 4HN; ${ }^{2}$ Senior Research Fellow, ${ }^{3}$ Director of the Effective Dental Practice Programme, Dental Health Services Research Unit, University of Dundee, The Mackenzie Building, Kirsty Semple Way, Dundee, DD2 4BF; ${ }^{5}$ Research and Development Manager, Scottish Dental Clinical Effectiveness Programme, Dundee Dental Education Centre, Frankland Building, Smalls Wynd, Dundee, DD1 4HN

*Correspondence to: Mr Simon Shepherd

Email: s.d.shepherd@dundee.ac.uk
}

Online article number E14

Refereed Paper - accepted 7 April 2011

DOI: 10.1038/sj.bdj.2011.822

${ }^{\circ}$ British Dental Journal 2011; 211: E14
Institute for Health and Clinical Excellence have developed guidelines to help clinicians recognise and tackle excessive alcohol consumption in attending patients. ${ }^{14,15}$ The focus of these guidelines is on the provision of advice by general medical practitioners; however general dental practitioners (GDPs) are potentially in an ideal position to identify excessive alcohol consumption and to provide ARA, particularly since alcohol is a well-established primary aetiological risk factor in the development of oral cancer. ${ }^{16-19}$ Nevertheless, there is a relative dearth of information about whether GDPs currently provide ARA..$^{20,21}$ Therefore the first aim of this study is to determine whether GDPs in Scotland currently provide ARA.

The second aim is to inform the development of an intervention to encourage GDPs to provide alcohol-related advice, should one be required. The focus is on understanding GDPs' intention to provide alcohol-related advice since intention to perform a behaviour has been shown to be a reliable indicator of actual performance. ${ }^{22-24}$

In order to achieve this aim we assessed beliefs derived from two psychological models, the Theory of Planned Behaviour (TPB) and Social Cognitive Theory (SCT). ${ }^{22,25}$ These specific theories were chosen because they explain behaviour in terms of beliefs that are amenable to change, they have been rigorously evaluated in other healthcare settings and have good evidence of predictive value for health relevant behaviours for healthcare professionals as well as patients. ${ }^{26-29}$

The TPB predicts that an individual is more likely to provide ARA if they have high intention to do so, if they think it will be easy for them to do (high perceived behavioural control), if they believe that doing so will result in consequences that are valued (positive attitude) and if the individual believes that people they respect would want them to perform that behaviour (positive subjective norm). SCT predicts that an individual is more likely to undertake a behaviour if they are confident in their performance ability (high self-efficacy).

Given that knowledge regarding the definition of excessive alcohol consumption and associated risk may influence whether GDPs provide advice, knowledge was also assessed as a possible predictive variable. 
Additionally, it is plausible to posit that GDPs' own alcohol-related behaviour may influence whether they provide ARA to patients; so a measure of personal alcohol consumption was also included in this study.

The research questions are:

1. Do GDPs in Scotland currently provide alcohol-related health advice?

2. What beliefs might predict GDPs' intention to provide alcohol-related health advice in primary care?

\section{METHODS}

The current study was a cross-sectional postal survey. Participants were GDPs randomly selected from across Scotland.

All general dental practitioners from a database containing details of NHS dentists practising in Scotland were assigned a number through computer random number generation. These random numbers were placed in numerical order from the smallest to the largest, and the first 300 practitioners selected to receive an invitation to participate in this study.

\section{Ethical considerations}

The Fife, Forth Valley \& Tayside Research Ethics Service on behalf of the Research Ethics Service Office considered the study to be an anonymous invitational dental service audit and formal ethical review was not required.

\section{Measures}

The measures assessing theoretical variables were created by following established principles and procedures. ${ }^{23,30,31}$

Preliminary work involving exploratory semi-structured interviews with a convenience sample of GDPs determined the salient views on the identification of alcohol misuse and provision of ARA in primary care and these results informed the development of the questionnaire items for this study. ${ }^{32}$

Unless otherwise stated, all measures were scored on seven-point scales.

\section{Outcome measures}

\section{Current behaviour}

GDPs were asked whether ARA was delivered in the preceding ten working days ('In the last ten working days have you provided alcohol-related advice? - yes/ no'). Ten working days was an arbitrary cut-off point determined by the authors as an appropriate time for this behaviour to have occurred at least once, given the evidence base. ${ }^{14,15}$

\section{Intention}

Two items assessed intention to provide alcohol advice: 'I intend to provide alcohol-related advice as part of patient management' and 'In general I have a plan about when I would provide alcohol advice as part of patient management'; the total intention score was a sum of these two items with higher scores reflecting greater overall intention. Possible scores ranged from 2-14.

\section{Predictive measures}

\section{Attitude}

1. 'Attitude indirect' included all 11 possible consequences to providing ARA identified in the preliminary study of GDPs: it would benefit the patient; improve the patients' oral cancer awareness; embarrass patients; upset patients; result in a more thorough examination; ensure patients' healthcare needs are appropriately managed; properly fulfil their role as a dentist; or improve professional relationships with patients. ${ }^{32}$ Participants were asked how strongly they agreed with each of these consequences and how important they were. Each consequence score was multiplied by the corresponding evaluation score. The total score was the mean of the multiplicative scores. Possible scores ranged from 1 to 49

2. 'Attitude direct' was the mean score of eight general attitude items: 'I think providing alcohol-related advice is: not useful-useful; not embarrassingembarrassing; something I am not confident to do-something I am confident I can do; not practicalpractical; not interesting-interesting; not relevant-relevant; not beneficialbeneficial; something I do not want to do-something I really want to do'.

\section{Perceived behavioural control (PBC)}

1. 'PBC indirect' was the mean score of 13 items relating to possible barriers to determining alcohol problems, to providing ARA, and to managing patients identified in the preliminary study: 'In relation to providing alcohol-related health advice how difficult do you find it to: talk to an embarrassed patient; find the time to include the advice during a consultation; know what to say; provide related literature; effectively influence what patients do?' 'How difficult do you find it to: determine the alcohol intake of your patients; record the alcohol intake of your patients; identify at-risk drinking behaviour; refer a patient to a general medical practitioner; refer a patient to a consultant for alcohol addiction; refer a patient to a telephone helpline; follow available guidelines; undertake training on alcohol-related matters?' Participants were asked to rate how difficult it would be for them to overcome these barriers ('not at all difficult' to 'extremely difficult'). All items were scored with higher scores reflecting greater perceived behavioural control (less difficulty in overcoming each barrier)

2. 'PBC direct' was the mean score of four general items related to barriers to providing ARA: 'I would like to provide alcohol-related advice but don't really know if I can; whether I provide alcohol-related advice is entirely up to me; I can provide alcohol-related advice if I really wanted to' and 'I think providing alcohol advice is difficult'

3. 'PBC general advice' was the score from a single item related to selfreport difficulty in providing any type of advice: 'In general I find it difficult to give any type of advice to patients'.

\section{Subjective norm}

1. The indirect measure of subjective norm included seven items assessing whether they felt motivated to comply the people identified in the preliminary study as putting pressure on them to provide ARA. These were 'the General Dental Council; the British Dental Association; Scottish Intercollegiate Guidelines Network; Scottish Dental Clinical Effectiveness Programme; National Institute for 
Health and Clinical Excellence; colleagues; and patients.' 'SN indirect' was the mean of seven multiplicative scores of these items

2. Direct subjective norm 'SN direct' was the score of a single self-report item regarding pressure to provide alcoholrelated advice: 'I feel under pressure to provide alcohol advice'.

\section{Self-efficacy}

1. 'Self-efficacy alcohol advice' was assessed with eight items: 'How confident are you that you can: determine the alcohol intake of your patients; record the alcohol intake of your patients; identify at-risk drinking behaviour; refer a patient to a general medical practitioner; refer a patient to a consultant for alcohol addiction; refer a patient to a telephone helpline; follow available guidelines; undertake training on alcohol-related matters?'. The total score was the mean of the sum of these items, with higher scores reflecting greater self-efficacy (greater confidence) for providing alcohol related advice

2. 'Self-efficacy general advice' was assessed with six items related to confidence in providing advice in general: 'How confident are you that you: can talk to a patient about a sensitive topic; can find the time to include the advice during a consultation; will know what to say; provide related literature; can effectively influence what patients do; discuss issues that you find embarrassing with patients?'

The total self-efficacy score for general advice was the mean of the sum of these items, with higher scores reflecting greater self-efficacy for providing any advice to patients.

\section{Knowledge}

Knowledge was assessed with ten items, four relating to the maximum safe amount of alcohol consumed in a single session for standard drinks of beer, wine, spirits or alco-pops and six items on the relationship between alcohol and health outcomes such as general health, oral health, oral cancer, smoking and oral cancer, caries and periodontal disease. Each correct answer was given a score of one. Possible scores ranged from zero to ten.

\section{Personal behaviour}

This was assessed using the Alcohol Use Disorders Identification Tool (AUDIT). ${ }^{33}$ This is a ten-item questionnaire with a possible score of 40 . Higher total scores reflect higher tendency towards alcoholrelated harm or dependence: scores below 8 indicate low harm, in the range 8-15 represent a medium or hazardous alcohol problem, 16 or greater represents an increased level of harm, and scores above 20 warrant further investigation and evaluation for dependence. ${ }^{33,34}$

\section{Demographics}

Demographic variables included time qualified as a dentist; gender; number of clinical sessions (0.5 days) worked on average per week; total practice list size; if the practice was rural and/or remote; the number of other clinicians in the practice and whether the respondent was a vocational trainer.

\section{Power calculation and sample size}

A priori power analysis showed that a sample size of 178 would be required to achieve a medium effect size $=0.15$ with $95 \%$ power based on a critical $\mathrm{F}=1.85$ using a multiple regression statistical test with 11 predictors (attitude direct/ indirect, perceived behavioural control direct/indirect/general advice, subjective norm direct/indirect, self-efficacy alcohol advice/general advice, knowledge, and personal behaviour).

\section{Procedure}

Previous surveys in this population have achieved response rates of approximately $60 \%$. Therefore, questionnaires were sent to a random sample of 300 GDPs working in the general dental services. A follow-up reminder letter with a duplicate questionnaire was sent three weeks after the first and followed subsequently with postcard reminder at six weeks.

\section{Statistical analysis}

Ten percent of data was randomly selected for double entry with descriptive statistics completed to ensure consistency and accuracy of data entry. Statistical significance was based on two-sided tests with $p<0.05$ as the criterion. Missing data for each questionnaire item was replaced with the individual's mean over all the items of that measure, providing only two or fewer items from that measure were missing.

The data was examined for univariate outliers using z scores $>3.29$ ( $p=0.001)$ and multivariate outliers using residuals scores, with a criterion of Mahalanobis distance at $\mathrm{p}<0.001$. Variables were examined for their approximation to a normal distribution using skewness and kurtosis statistics with cut-off greater than \pm 1 . Measures were tested for internal consistency using Cronbach's alpha. The relationship between the predictive variables and the outcome variable of intention to provide ARA was examined using Pearson's correlation analysis and multiple regression analysis. Multicollinearity was tested by screening for correlations between predictive variables greater than 0.90 , low tolerance (1-SMC) and high standard errors (relative to the scale of each variable) for regression coefficients. ${ }^{35}$

\section{RESULTS}

\section{Response rate and participants}

Out of the 300 questionnaires posted, six were returned undeliverable with 175 completed and returned giving a response rate of $60 \%$ (175/294). The final sample profile was: 60\% (104/172) were male, qualified, on average, for 19 years ( $S D=9.1$ ), worked full-time (mean (SD) sessions per week $=9$ (2.26)), with a practice list size of 2,081 $(\mathrm{SD}=1,351)$. Eleven percent $(18 / 172)$ were vocational trainers and 24\% (42/172) considered their practice to be remote and/or rural. The average number of other dentists in the practice was three, ranging from zero to 13. Eighty-three percent (142/171) worked in general dental practice, 13\% $(22 / 171)$ in salaried services, 5\% (8/171) in community dental services and 1\% (2/171) in hospital-based services. Ninety-two percent (158/171) worked in only one service while $8 \%(13 / 171)$ reported working in two or more services.

\section{Data}

No outliers were identified. All variables showed psychometrically acceptable levels of skewness or kurtosis. There was no evidence of multicollinearity: the highest Pearson correlation was $r=0.65$ between 
self-efficacy alcohol advice and PBC indirect; the collinearity diagnostics showed the tolerance values for all variables included in the models were over 0.6 and the standard errors of the regression coefficients were all less than 0.1 .

\section{Do GDPs currently provide alcohol-related advice?}

Eighty-three percent (142/172) had not provided advice in the previous ten working days.

\section{What beliefs might predict GDPs' intention to provide alcohol-related health advice in primary care?}

On average, GDPs have low intention to provide ARA (mean 3.57; SD = 1.40).

Descriptive statistics for the predictive variables assessed in this study are shown in Table 1 . The results suggest that participating GDPs do not have a positive attitude to providing ARA, believe that providing ARA is difficult and also have low self-efficacy in providing ARA in primary care. Additionally, in general they do not feel under pressure to provide ARA. With an average score of 3 out of 10 , GDPs showed relatively poor knowledge on recommended alcohol consumption guidelines and associated risk. Personal alcohol consumption, as assessed by the AUDIT, was generally below the accepted threshold score of 8 for harmful alcohol consumption, with $85 \%$ scoring less than 8 , approximately $14 \%$ exhibiting moderate levels of harm and one respondent scoring in the high alcohol harm range bordering on dependence.

Intention to provide alcohol advice was significantly related to attitude indirect/ direct, $\mathrm{PBC}$ indirect, $\mathrm{PBC}$ general advice, subjective norm indirect/direct and selfefficacy alcohol advice/general advice. Each of these variables were entered into a stepwise multiple regression with intention as the dependent variable (Table 2). Attitude direct/indirect, self-efficacy general advice, and subjective norm indirect explained 35\% of the variance in intention to provide ARA.

Individual items from these variables were then entered into a further exploratory stepwise multiple regression equation (Table 3). Five items explained $41 \%$ of the variance in intention: 'I think providing

Table 1 Descriptive statistics and Pearson correlations of predictive measures and behavioural intention

\begin{tabular}{|c|c|c|c|c|}
\hline \multirow{2}{*}{ Predictive measures } & \multicolumn{3}{|c|}{ Descriptive statistics } & \multirow{2}{*}{$\begin{array}{l}\begin{array}{l}\text { Pearson's } \\
\text { correlation }\end{array} \\
\text { Intention }\end{array}$} \\
\hline & Alpha & Range & Mean (SD) & \\
\hline Intention & 0.76 & $2-14$ & $3.57(1.40)$ & 1.00 \\
\hline Attitude indirect & 0.85 & $1-44$ & $22.94(6.87)$ & $0.54^{* *}$ \\
\hline Attitude direct & 0.86 & $1-7$ & $4.30(1.04)$ & $0.52^{* *}$ \\
\hline PBC indirect & 0.83 & $1-7$ & $3.59(0.93)$ & $0.37^{* *}$ \\
\hline PBC direct & 0.31 & $1-7$ & $4.17(0.88)$ & $-0.05 \mathrm{~ns}$ \\
\hline PBC general advice & $\mathrm{n} / \mathrm{a}$ & $1-7$ & $5.09(1.77)$ & $0.18^{*}$ \\
\hline Subjective norm indirect & 0.87 & $1-30$ & $12.77(6.70)$ & $0.35^{* *}$ \\
\hline Subjective norm direct & $\mathrm{n} / \mathrm{a}$ & $1-7$ & $2.24(1.37)$ & $0.18^{*}$ \\
\hline Self-efficacy general advice & 0.81 & $1-7$ & $3.79(1.03)$ & $0.45^{* *}$ \\
\hline Self-efficacy alcohol advice & 0.79 & $1-7$ & $3.65(1.05)$ & $0.35^{* *}$ \\
\hline Knowledge & 0.74 & $1-9$ & $3.43(1.98)$ & $-0.05 \mathrm{~ns}$ \\
\hline Personal alcohol behaviour & $\mathrm{n} / \mathrm{a}$ & $0-19$ & $4.71(3.09)$ & $0.05 \mathrm{~ns}$ \\
\hline \multicolumn{5}{|c|}{ 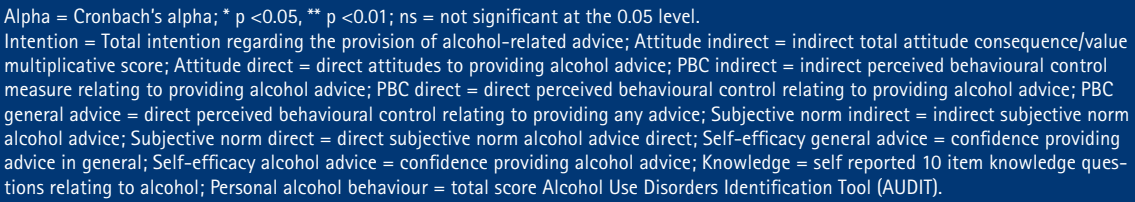 } \\
\hline
\end{tabular}

Table 2 Results of explorative stepwise regression analysis of variables predicting intention to provide alcohol-related advice

\begin{tabular}{|c|c|c|c|c|c|c|}
\hline Entered & B & $\beta$ & $R^{2}$ change & Adj. $R^{2}$ & $\mathrm{~d} f$ & $\mathrm{~F}$ \\
\hline Attitude direct ARA & 0.25 & $0.19^{*}$ & 0.25 & & & \\
\hline Attitude indirect ARA & 0.05 & $0.24^{* *}$ & 0.05 & & & \\
\hline Self-efficacy deneral advice & 0.29 & $0.22^{* *}$ & 0.03 & & & \\
\hline Subjective norm indirect ARA & 0.04 & 0.19 & 0.03 & 0.35 & 4,157 & $22.56^{* * *}$ \\
\hline \multicolumn{7}{|c|}{$\begin{array}{l}\text { Outcome: behavioural intention } \\
\text { Variables included in the exploratory stepwise regression equation: attitude indirect, attitude direct, perceived behavioural control indirect, } \\
\text { perceived behavioural control general advice, subjective norm indirect, subjective norm direct, self-efficacy general advice, self-efficacy alcohol } \\
\text { advice. Note: Adjusted } R^{2} \text { for this model is } 0.35 ; F=22.56(0.001) ; B=\text { unstandardised coefficient; } \beta=\text { standardised coefficient; }{ }^{*} p<0.05 ;{ }^{* *} \\
p<0.01 ;\end{array}$} \\
\hline
\end{tabular}

alcohol-related advice is practical'; 'I am confident I can provide related literature'; 'My providing alcohol advice is likely to cause embarrassment'; 'I feel under pressure from colleagues to provide advice and am motivated to do what my colleagues think I should'; and 'I think providing alcohol related advice is beneficial'.

Neither knowledge nor personal alcohol-related behaviour (the dentist's own drinking habits) were significantly related to intention to provide ARA.

\section{DISCUSSION}

The first aim of this study was to determine if GDPs currently provide ARA. Few participating GDPs currently provide ARA, suggesting that there is scope to improve this behaviour. This result is consistent with previous studies with GDPs in the United Kingdom which incorporated alcohol-related issues. ${ }^{20,36,37}$

The second aim of this study was to identify beliefs that predict intention to provide ARA to inform the development of an intervention to encourage the delivery of advice. All of the beliefs derived from the psychological theories significantly predicted intention to provide ARA and acted in accordance with theoretical expectations in that more positive attitude, higher subject norm, greater perceived 
Table 3 Results of explorative stepwise regression of the individual questionnaire items explaining variance in intention to provide alcohol-related advice

\begin{tabular}{|c|c|c|c|c|c|c|}
\hline Entered & B & $\beta$ & $R^{2}$ change & Adj. $R^{2}$ & $\mathrm{~d} f$ & $\mathrm{~F}$ \\
\hline 1. Providing ARA is practical & 0.16 & 0.17 & 0.22 & & & \\
\hline $\begin{array}{l}\text { 2. Confidence in providing } \\
\text { related literature }\end{array}$ & 0.28 & $0.26^{* *}$ & 0.08 & & & \\
\hline $\begin{array}{l}\text { 3. Importance of not being } \\
\text { embarrassed in front of patients }\end{array}$ & 0.03 & $0.22^{* *}$ & 0.06 & & & \\
\hline $\begin{array}{l}\text { 4. Feeling under pressure from } \\
\text { colleagues to provide ARA }\end{array}$ & 0.04 & $0.22^{* *}$ & 0.04 & & & \\
\hline 5. Providing ARA is beneficial & 0.21 & $0.20^{*}$ & 0.03 & 0.41 & 5,98 & $15.34^{* * *}$ \\
\hline \multicolumn{7}{|c|}{ 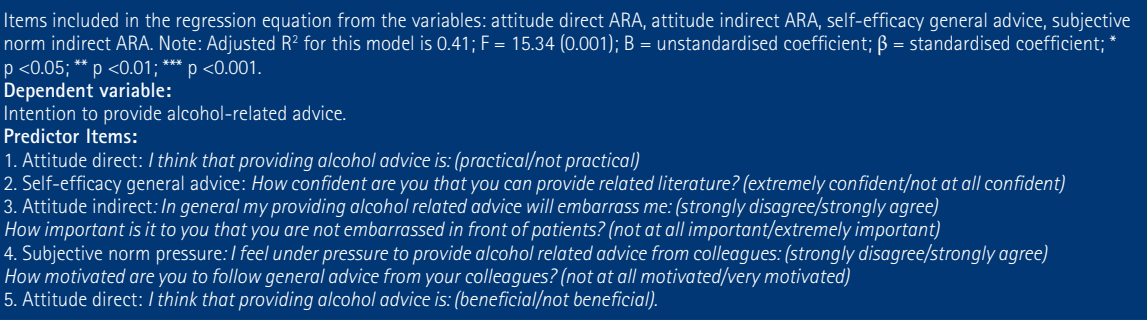 } \\
\hline
\end{tabular}

behavioural control and self-efficacy all were associated with greater intention to provide alcohol-related advice.

A stepwise regression analysis explored the relative importance of each of the predictive variables in accounting for the variance in intention to provide ARA (Table 2). Attitude (direct/indirect), selfefficacy general advice and subjective norm (indirect) accounted for 35\% of the variance in intention.

An item analysis was then performed to identify the pivotal items within these predictive variables (Table 3). Only five items accounted for $41 \%$ of the variance in intention $(\mathrm{F}(5,98)=15.34, \mathrm{p}<0.001)$ : 'I think providing alcohol related advice is practical' ( $\beta=0.17$ ), 'I am confident about providing related literature' $(\beta=0.26)$, 'Providing alcohol advice is not likely to cause me embarrassment' ( $\beta=0.22)$, 'I feel under pressure to provide alcohol advice from colleagues' ( $\beta=0.22)$ and 'I think providing alcohol related advice is beneficial' $(\beta=0.20)$. The results of the item analysis suggest that GDPs may be encouraged to provide ARA by an intervention which addresses these specific issues.

Knowledge of recommended sensible alcohol consumption guidelines was relatively poor, although this did not seem to be an issue as knowledge was not related to intention to provide ARA. This is consistent with previous work suggesting that knowledge alone is usually not sufficient to influence behaviour and that implementation effectiveness is often reliant on factors other than knowledge. ${ }^{38-40}$ Personal alcohol behaviour also was not associated with intention to provide ARA. These results suggest that an intervention which targets either of these variables is unlikely to encourage GDPs to provide ARA.

\section{CONCLUSION}

The results of this study suggest that there is scope to increase the provision of alcohol-related health advice in primary care dentistry. This study also identified some possible targets of an intervention to encourage GDPs to provide alcoholrelated advice in primary dental care. The next phase is to develop and evaluate an intervention to encourage GDPs to provide ARA, based on the results of this study.

We would like to thank the Scottish Dental Practice Based Research Network (SDPBRN) team who supported and funded this study; and the University of Dundee.

1. Anderson P, Baumberg B. Alcohol and public health in Europe. Short report. London: Institute of Alcohol Studies UK, 2007.

2. Room R, Babor T, Rehm J. Alcohol and public health. Lancet 2005; 365: 519-530.

3. World Health Organisation. WHO Expert Committee on Problems Related to Alcohol Consumption. Second report. Geneva: World Health Organisation, 2007.

4. Rehm J, Taylor B, Patra J. Volume of alcohol consumption, patterns of drinking and burden of disease in the European region 2002. Addiction 2006; 101: 1086-1095.

5. Audit Scotland. Drug and alcohol services in Scotland. Report. Edinburgh: Audit Scotland, 2009.

6. Health Economics Unit, ASDHD, Scottish Government. Costs of alcohol use and misuse in Scotland. Edinburgh: The Scottish Government, 2008.

7. NHS National Services Scotland. Alcohol statistics Scotland 2009. Edinburgh: ISD Publications, 2009. 8. Ballesteros J, Duffy J C, Querejeta I, Arino J,
Gonzalez-Pinto A. Efficacy of brief interventions for hazardous drinkers in primary care: systematic review and meta-analyses. Alcohol Clin Exp Res 2004; 28: 608-618.

9. Beich A, Gannik D, Malterud K. Screening and brief intervention for excessive alcohol use: qualitative interview study of the experiences of general practitioners. BMJ 2002; 325(7369): 870B-872B.

10. Beich $A$, Thorsen $T$, Rollnick S. Screening in brief intervention trials targeting excessive drinkers in general practice: systematic review and metaanalysis. BMJ 2003; 327(7414): 536-542.

11. Cuijpers $P$, Riper $H$, Lemmers $L$. The effects on mortality of brief interventions for problem drinking: a meta-analysis. Addiction 2004; 99: 839-845.

12. Kaner E F, Beyer F, Dickinson $\mathrm{H} O$ et al. Effectiveness of brief alcohol interventions in primary care populations. Cochrane Database Syst Rev 2007; (2): CD004148.

13. Richmond R, Heather N, Wodak A, Kehoe L, Webster I. Controlled evaluation of a general practice-based brief intervention for excessive drinking. Addiction 1995: 90: 119-132.

14. National Institute for Health and Clinical Excellence Alcohol use disorders: preventing the development of hazardous and harmful drinking. NICE public health guidance 24. London: NICE, 2010.

15. Scottish Intercollegiate Guidelines Network. The management of harmful drinking and alcohol dependence in primary care: a national clinical guideline. Edinburgh: Scottish Intercollegiate Guidelines Network, 2003

16. Carter L M, Ogden G R. Oral cancer awareness of general medical and general dental practitioners. Br Dent J 2007; 203: E10.

17. Hashibe M, Brennan P, Benhamou S et al. Alcohol drinking in never users of tobacco, cigarette smoking in never drinkers, and the risk of head and neck cancer: pooled analysis in the International Head and Neck Cancer Epidemiology Consortium. J Natl Cancer Inst 2007; 99: 777-789.

18. Petti S. Lifestyle risk factors for oral cancer. Oral Oncol 2009; 45: 340-350.

19. Warnakulasuriya S. Global epidemiology of oral and oropharyngeal cancer. Oral Oncol 2009; 45: 309-316.

20. Dyer T A, Robinson P G. General health promotion in general dental practice - the involvement of the dental team. Part 2: a qualitative and quantitative investigation of the views of practice principals in South Yorkshire. Br Dent J 2006; 201: 45-51.

21. Dyer T A, Robinson P G. General health promotion in general dental practice - the involvement of the dental team. Part 1: a review of the evidence of effectiveness of brief public health interventions. Br Dent J 2006; 200: 679-685.

22. Ajzen I. The theory of planned behaviour. Organ Behav Hum Decis Process 1991; 50: 179-211.

23. Conner M, Norman P. Predicting health behaviour: research and practice with social cognition models. 2nd ed. Maidenhead: Open University Press, 2005.

24. Eccles M P, Hrisos S, Francis J et al. Do self-reported intentions predict clinicians' behaviour: a systematic review. Implement Sci 2006; 1: 28.

25. Bandura A. Health promotion from the perspective of social cognitive theory. Psychol Health 1998; 13: 623-649.

26. Bonetti $D$, Eccles $M$, Johnston $M$ et al. Guiding the design and selection of interventions to influence the implementation of evidence-based practice: an experimental simulation of a complex intervention trial. Soc Sci Med 2005; 60: 2135-2147.

27. Bonetti D, Johnston M, Pitts N B et al. Can psychological models bridge the gap between clinical guidelines and clinicians' behaviour? A randomised controlled trial of an intervention to influence dentists' intention to implement evidence-based practice. Br Dent J 2003; 195: 403-407.

28. Bonetti D, Pitts N B, Eccles M et al. Applying psychological theory to evidence-based clinical practice: identifying factors predictive of taking intra-oral radiographs. Soc Sci Med 2006; 63: 1889-1899.

29. Walker A E, Grimshaw J M, Armstrong E M. Salient beliefs and intentions to prescribe antibiotics for 
patients with a sore throat. Br J Health Psychol 2001; 6: 347-360.

30. Azjen I. Constructing a TPB questionnaire: conceptual and methodological considerations. Available at http://people.umass.edu/ aizen/pdf/tpb.measurement.pdf (accessed 19 September 2011).

31. Francis J J, Eccles M P, Johnston M et al. Constructing questionnaires based on the theory of planned behaviour. A manual for researchers. Newcastle: Centre for Health Services Research, 2004

32. Shepherd S, Young L, Clarkson J, Bonetti D, Ogden $G$ R. General dental practitioner views on providing alcohol related health advice; an exploratory study. Br Dent J 2010; 208: E13.

33. Babor T F, Higgins-Biddle J C, Saunders J B, Monteiro M G. AUDIT - the Alcohol Use Disorders Identification Test. Guidelines for use in primary care. 2nd ed. Geneva: World Health Organisation 2001.

34. Fiellin D A, Reid M C, O'Connor P G. Screening for alcohol problems in primary care: a systematic review. Arch Intern Med 2000; 160: 1977-1989.

35. Tabachnick B G. Fidell L S. Using multivariate statistics. 4th ed. Needham Heights, MA: Allyn \&t Bacon, 2001.

36. Brocklehurst P R, Baker S R, Speight P M. A qualitative study examining the experience of primary care dentists in the detection and management of potentially malignant lesions. Mechanics of the referral and patient communication. Br Dent J 2010; 208: E4.

37. Warnakulasuriya K A, Johnson N W. Dentists and oral cancer prevention in the UK: opinions, attitudes and practices to screening for mucosal lesions and to counselling patients on tobacco and alcohol use: baseline data from 1991. Oral Dis 1999; 5: 10-14.

38. Eccles M, Grimshaw J, Walker A, Johnston M, Pitts $\mathrm{N}$. Changing the behaviour of healthcare professionals: the use of theory in promoting the uptake of research findings. J Clin Epidemio/ 2005; 58: 107-112.

39. McGlone P Watt R, Sheiham A. Evidence-based dentistry: an overview of the challenges in changing professional practice. Br Dent J 2001; 190: $636-639$

40. Nilsen $\mathrm{P}$, Aalto M, Bendtsen $\mathrm{P}$, Seppa K.

Effectiveness of strategies to implement brief alcohol intervention in primary healthcare - a systematic review. Scand J Prim Health Care 2006; 24: $5-15$ 